\title{
AOR
}

Selected Papers of \#AolR2019:

The $20^{\text {th }}$ Annual Conference of the Association of Internet Researchers Brisbane, Australia / 2-5 October 2019

\section{VIDEOGAME ANALYTICS, SURVEILLANCE CAPITALISM, AND THE RETENTIONAL ECONOMY OF PLAY}

\author{
Ben Egliston, University of Sydney
}

\begin{abstract}
Data analytics tools are increasingly prevalent in videogames and are reliant on the surveillant capture and relay of user data. In this paper I present some conceptual work and preliminary analysis of the analytics tool 'DotaPlus' used in Dota 2. Through my analysis, I frame DotaPlus as a site of 'surveillance capitalism', using data derived from various modes of surveillance to generate potentials for commercially desirable gameplay experience.
\end{abstract}

\section{Introduction}

Recently, Zuboff has argued that data derived from contemporary forms of technological surveillance have the capacity to offer significant knowledge about people's behavior, and the potential to profoundly shape that behavior in future (2019). Within the context of contemporary global technoculture, Zuboff argues that the surveillant collection of data about people's lives are put in service of capitalism. Data facilitate the continued use and development of technologies (see also Srnicek, 2017), but are also traded commodities valuable in the development of systems of prediction and probabilization (which as Zuboff and many others see as creating subjects more amenable to capitalism). In short, the power and control exerted through logics of accumulation and the technologies and techniques of surveillance denote what Zuboff calls 'surveillance capitalism'.

In this paper, I present initial analyses of videogame analytics tools as a site of surveillance capitalism. Particularly, I focus on the tool 'DotaPlus' for the popular multiplayer game Dota 2. DotaPlus' various features are enabled through the monitoring of user activity. Distinct from Zuboff, who argues that sites of modern surveillance capitalism are in large part tied to the 'behavioral futures' marketplace, in this paper I suggest that the power and control exerted over subjects, as to make them more Suggested Citation (APA): Egliston, B. (2019, October 2-5). Videogame analytics, surveillance capitalism, and the retentional economy of play. Paper presented at AoIR 2019: The $20^{\text {th }}$ Annual Conference of the Association of Internet Researchers. Brisbane, Australia: AolR. Retrieved from http://spir.aoir.org. 
amenable to capitalism, operates by using surveillance and data accumulation as a technique for directly shaping how people experience, feel and think about the consumption of a particular product (here, Dota 2).

In order to develop this argument, I draw from Stiegler's concept of retentional economy (2010, pp. 8-10). For Stiegler, retentional economy broadly describes how memory and human experience are retained in, and transmitted through, material objects or environments (what he calls tertiary retention). This process changes up the temporal structure of humans' phenomenological experience - that is, for Stiegler, how people remember their pasts, and develop forward-leaning expectations of the future. As Stiegler sees it, new kinds of tertiary retentions condition human experience increasingly, within the context of modern, commercial technics, to generate economic value (a dynamic he calls 'psychopower'). Drawing from Stiegler, the claim made in this paper is that the way people play and experience Dota 2 is transformed through analytics as a form of tertiary retention; the numerical and statistical quantification of play, enabled by various modes of surveillance, transforming how players affect and are affected by Dota 2, producing qualities that might be seen as economically desirable for Valve.

\section{Methods}

From March 2018 (DotaPlus launch) until January 2019, I analyzed material from 40 threads on the Dota 2 subreddit, '/r/Dota2' and 15 videos (from YouTube and Twitch). I focused on content about DotaPlus and play experience. Empirical material presented represents a convenient sample. In line with the exploratory nature of the research, data was first open coded to determine themes, and then relations between themes were established. Concepts derived from the literature on data, surveillance and humantechnology relations sensitized me to lines of inquiry in analysis. My own experience as a DotaPlus user over this period (340 Dota 2 matches, approximately 300 hours playtime) enabled me to parse and analyze the data.

\section{DotaPlus, surveillance, power}

DotaPlus is a paid subscription-based form of self-tracking software for the game Dota 2. DotaPlus features various forms of data analytics to do with players' performance in different aspects of the game. To illustrate how DotaPlus might create the potential for continued consumption of the game, I focus on data to do with the 'positive' impacts of DotaPlus on the phenomenal quality of playing the game (cf. Ash, 2015). Each of DotaPlus' features involve the collection, sorting, and presentation of player data in some sort of numerical or visual format. I found that DotaPlus' features could be classified according to three different modes of surveillance: self-surveillance, lateral surveillance and autonomous, nonhuman surveillance - each of which with various effects.

Self-surveillance in DotaPlus involves the real-time scoring of players' ingame performance. One feature compares player performance to an average of similarly skilled players (see figure 1). Across the empirical material, this self-tracking feature 
was widely seen as productive - players performing aspirationally (with the potential for positive affects when the goal is met) and negotiating the game through their own somatic and cognitive faculties and the gaze of the data. Self-tracking features also include the stylized quantification of gameplay maneuvres (e.g. in flashing and glowing numbers over a player's character), with some users pointing out the emotional and affective connections they had to this data (over using it for skill development).

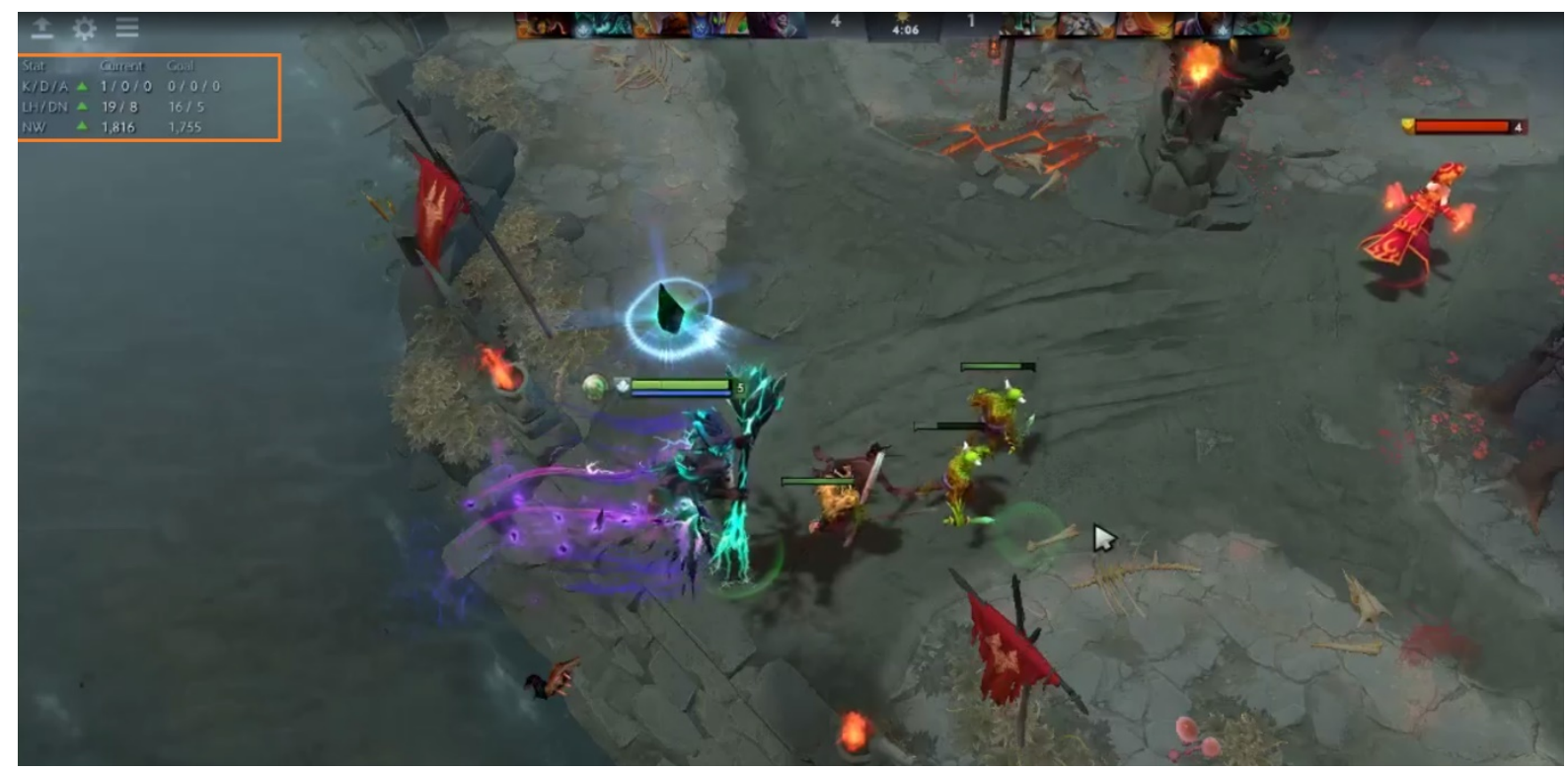

Figure 1 - Self-tracking in DotaPlus

Lateral (or 'peer') surveillance (see Andjrevic, 2004) afforded by DotaPlus is evident in the 'game summary' feature - which enable the surveillance of player performance over a game. Across the material here, a key theme was the capacity to draw on and reflect upon past performance, exteriorized in the form of data, potentially shaping future encounters with the game. Others saw it as 'amplifying' the competition between players. One thread discussed affects of anxiety or dread about underperforming and anticipating being admonished by teammates (who can view the summary if they have DotaPlus), as a result, forcing players to play 'better'.

Lastly, I consider the autonomous surveillance that enable the tool's machine learning features. Through this collection of user data Valve provide dynamic systems of prediction which enroll players into anticipatory regimes in negotiating each match. Discussions situated this as a mode of experience of pre-empting experience, lessening the need for accumulating large amounts of subjective memory, assisting in negotiating a difficult and forbidding game.

\section{Conclusion}

In exploring these three sites of surveillance, which variously exteriorize gameplay performance as data, I argue that digital traces of player activity work as tertiary retention - captured and fed back to users, altering the experience of playing Dota 2 in 
a way that may be economically desirable for Valve. Reading Stiegler and Zuboff together, DotaPlus might productively be understood as a site of surveillance capitalism, shaping people's experience of playing the game through various features enabled by data from various forms of surveillance.

This paper is expected to be of interest to games and media researchers in spotlighting new techniques used by game developers to create subjects amenable to continued consumption. More broadly, it is pertinent in a moment where surveillance-based technologies mediate our everyday lives.

\section{References}

Ash, J. (2015). The interface envelope: Gaming, technology, power. New York, NY: Bloomsbury

Andrejevic, M. (2004). The work of watching one another: lateral surveillance, risk and governance. Surveillance and society, 2(4), 479- 497

Srnicek, N. (2017). Platform capitalism. Cambridge, UK: Polity.

Stiegler, B. (2010). For a new critique of political economy. Cambridge, UK: Polity.

Zuboff, S. (2019). The age of surveillance capitalism: The fight for a human future at the new frontier of power. New York, NY: Public Affairs. 